\title{
Compromiso gastrointestinal inusual en paciente pediátrico con vasculitis asociada a anticuerpos anticitoplasma de neutrófilos. Reporte de un caso
}

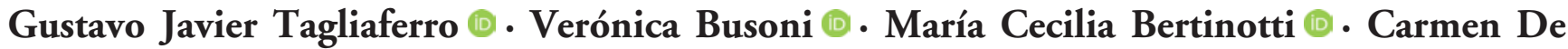 \\ Cunto (D) - Marina Orsi (D)
}

Hospital Italiano de Buenos Aires, Ciudad Autónoma de Buenos Aires. Argentina.

Acta Gastroenterol Latinoam 2021;51(4):437-440

Recibido: 13/04/2021 / Aceptado: 02/11/2021 / Publicado online: 13/12/2021 / bttps://doi.org/10.52787/USCW3486

\begin{abstract}
Resumen
Las vasculitis se caracterizan por presentar inflamación de la pared de los vasos sanguineos; un grupo de estas enfermedades son las asociadas a anticuerpos anticitoplasma de neutrófilos. Suelen ocurrir en la edad adulta y son poco comunes en la infancia. La enfermedad generalmente afecta el pulmón, el riñón y la piel, siendo infrecuente el compromiso gastrointestinal. Aqui se describe el caso de un paciente pediátrico con hemorragia digestiva secundaria a una vasculitis ANCA positivo. La endoscopía mostró lesiones eritematosas en forma parcheada y hematoma de pared a nivel de colon. Reportamos una condición clinica bastante infrecuente que puede presentar complicaciones. Creemos adecuado sospecharla ante todo compromiso intestinal en el que no sea claro el proceso inflamatorio subyacente, más aún cuando involucra otros órganos.
\end{abstract}

Palabras claves. Vasculitis; ANCA-p; colitis; hematoma de colon.

\section{Unusual Gastrointestinal Involvement in a Pediatric Patient with Anti-Neutrophil Cytoplasmatic Antibodies-Associated Vasculitis. A Case Report}

\section{Summary}

Vasculitis is characterized by presenting inflammation of the wall of blood vessels, one type of these diseases are those associated with anti-neutrophil cytoplasm antibodies. They usually occur in adulthood and are rare in childhood. The disease generally affects the lung, kidney, and skin, with gastrointestinal involvement being rare. Here we describe the case of a pediatric patient with gastrointestinal bleeding secondary to ANCA positive vasculitis. Endoscopy revealed patchy erythematous lesions and wall hematoma at the level of the colon. Although we report a fairly infrequent clinical condition, it is not without complications. We believe it is appropriate to suspect it, first of all, intestinal involvement where the underlying inflammatory process is not clear, even more so when it involves other organs.

Keywords. Vasculitis; p-ANCA; colitis; colon hematoma.

\author{
Abreviaturas \\ EANCA: Anti-citoplasma de neutrófilo. \\ GI: Gastrointestinal. \\ GPA: Granulomatosis con poliangeitis.
}

Correspondencia: Gustavo Javier Tagliaferro

Correo electrónico: gustavo.tagliaferro@hospitalitaliano.org.ar 
MPA: Poliangeítis microscópica.

EGPA: Granulomatosis eosinofilica con poliangeitis.

ANCA-C: Anticitoplasma de neutrófilo central.

ANCA-P: Anticitoplasma de neutrófilo periférico.

PR3: Proteinasa 3.

MPO: Mieloperoxidasa.

IRA: Insuficiencia renal aguda.

RNM: Resonancia magnética nuclear.

HD: Hemorragia digestiva.

\section{Introducción}

Las vasculitis sistémicas son un grupo de enfermedades que se caracterizan por presentar inflamación de la pared de los vasos sanguíneos. Esto determina una disminución del flujo sanguíneo, provocando isquemia y necrosis tisular, con posterior daño de órgano. Presentan una sintomatología variable y comúnmente se las puede clasificar, según el calibre del vaso comprometido, en grandes, medianas y pequeñas. ${ }^{1}$ Dentro de las vasculitis de pequeños vasos se encuentran las asociadas a anticuerpos anticitoplasma de neutrófilos (ANCA). Comprenden la granulomatosis con poliangeítis (GPA, Wegener's), la poliangeítis microscópica (MPA) y la granulomatosis eosinofílica con poliangeítis (EGPA, síndrome de ChurgStrauss). Es frecuente que el ANCA central (ANCA-C) reaccione contra proteinasa 3 (PR3) y que el ANCA periférico (ANCA-P) lo haga contra mieloperoxidasa (MPO). Caracterizadas como enfermedades sistémicas raras, suelen ocurrir en la edad adulta y son poco comunes en la infancia. Su patogenia no está del todo clara, se cree que los factores genéticos, ambientales y las anomalías en la respuesta inmune contribuyen a su desarrollo. En pediatría se reporta un mayor compromiso en niñas, y su inicio alcanza su punto máximo a principios de la segunda década. ${ }^{2}$ La presentación típica es la afectación aislada de uno o dos órganos con síntomas constitucionales y el diagnóstico se basa a menudo en la histopatología. Los órganos comúnmente involucrados son el pulmón, el riñón y la piel, siendo infrecuente el compromiso gastrointestinal (GI). La prevalencia de este último en pediatría varía de 0 a 5\% y los síntomas más comunes son la diarrea y el dolor abdominal. ${ }^{3,4}$ Debe tenerse en cuenta que si el compromiso GI se presenta en forma aislada dificulta muchas veces la sospecha diagnóstica. Debido a que estos trastornos son muy agresivos, el tratamiento temprano es fundamental para evitar resultados fatales. A continuación, reportamos un compromiso intestinal inusual en un paciente pediátrico, secundario a una MPA (vasculitis ANCA-p).

\section{Caso clínico}

Paciente femenino de 8 años de edad, previamente sana, derivada a nuestra institución para estudio y tratamiento por cuadro de insuficiencia renal aguda (IRA) con hematuria, proteinuria y anticuerpos ANCA +. Como antecedente, seis meses previos a la consulta, presentó decaimiento, vómitos y disminución del ritmo diurético. Se realizan múltiples estudios, entre los que se destaca la presencia de anemia, hematuria, proteinuria y anticuerpos ANCA +, por lo que se deriva a un centro de mayor complejidad. Al ingreso se constata oligoanuria e hiperkalemia, por lo cual inicia hemodiálisis. Se realiza biopsia renal que informa glomeruloesclerosis (global y difusa), proliferación extracapilar y nefropatía intersticial aguda difusa. Inmunofluorescencia: pauci-inmune. De acuerdo con el informe histológico y las serologías, se confirma diagnóstico de MPA (vasculitis ANCA-p). Inicia tratamiento con tres pulsos de metilprednisolona, con posterior descenso de corticoides por vía oral, sumando además azatioprina y diálisis peritoneal. A las doce horas de su egreso, presenta un episodio de proctorragia abundante. Al examen físico se encuentra hemodinámicamente estable, con abdomen blando depresible e indoloro y cánula de diálisis peritoneal sin signos de flogosis. Región perianal sin lesiones. Dentro de los exámenes complementarios, se realiza ecografía de abdomen en la que se observa dilatación de asa colónica y escaso líquido libre en cavidad abdominal. Se efectúa hemograma con hemoglobina $8,5 \mathrm{~g} / \mathrm{dl}$ y hematocrito $25 \%$. La cánula de diálisis muestra líquido claro, sin evidencia de sangrado. Durante el transcurso de la internación se constata caída de cinco puntos del hematocrito, por lo que recibe transfusión de glóbulos rojos y se realiza endoscopía digestiva. Se constata nodularidad y disminución de pliegues en segunda porción duodenal. La videoendoscopía muestra a nivel del colon lesiones parcheadas de aspecto vasculítico y hematoma de pared a $55 \mathrm{~cm}$ del margen anal (Figuras 1 y 2 ).

Por sospecha de compromiso gastrointestinal secundario a vasculitis se realizan biopsias, que son enviadas a anatomía patológica. Estas se realizan en forma escalonada de los sectores evaluados, siendo dirigidas en presencia de lesiones sugestivas. El procedimiento es bien tolerado por la paciente y sin complicaciones. En una de las muestras de duodeno se reconoce congestión vascular con extravasación eritrocitaria focal y vasculitis focal de vasos capilares (Figura 3). La muestra colónica presenta histoarquitectura glandular conservada, hallándose un foco sospechoso de inflamación perivascular con eosinófilos. Ante el hallazgo de compromiso extrarrenal de vasculitis activa, se suspende azatioprina y se inicia ciclofosfamida. Evoluciona en forma favorable, con posterior egreso hospitalario, y continúa controles ambulatorios con reumatología, nefrología y gastroenterología en nuestra institución. 
Figura 1. A) Duodeno nodular con pérdida de pliegues. B) Mucosa colónica con lesiones eritematosas de 3-5 milimetros. C) Lesión eritematosa de colon.
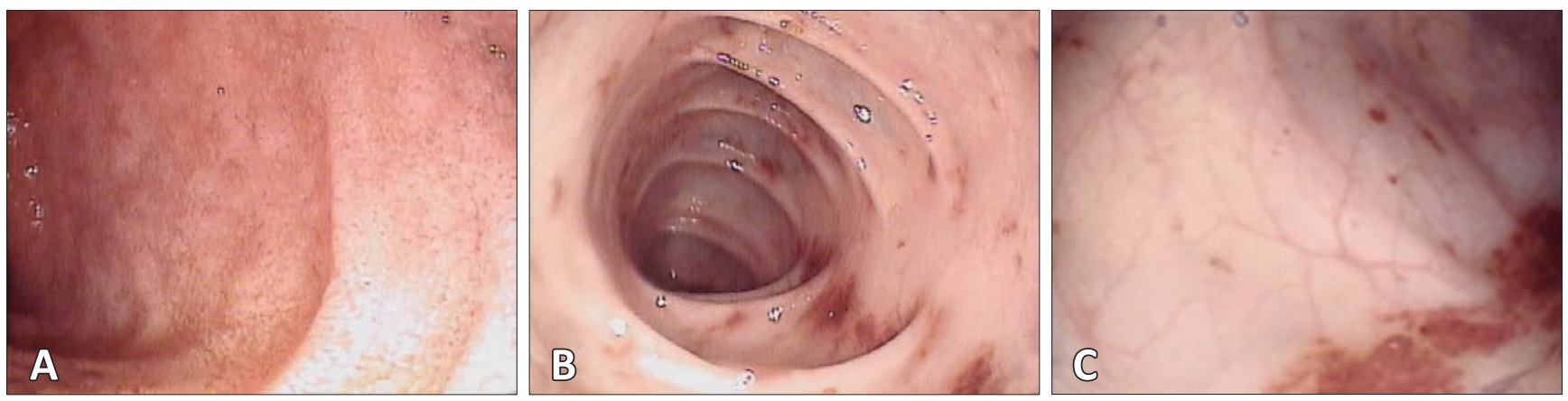

Figura 2. Colon sigmoideo; hematoma de pared que ocupa el $50 \%$ de la luz intestinal.
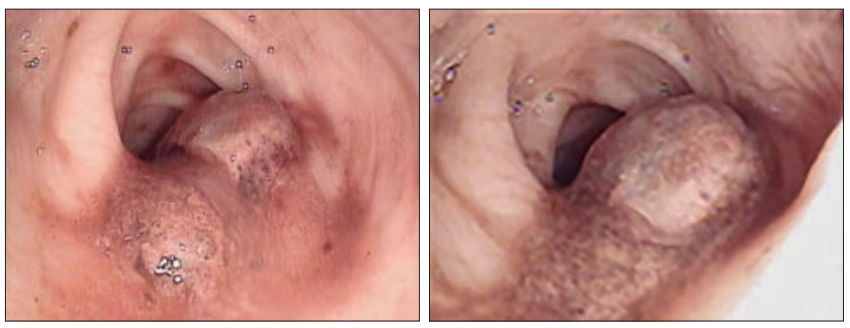

\section{Discusión}

Existen numerosos reportes publicados en población adulta sobre el compromiso gastrointestinal de la vasculitis ANCA-p, pero hay muy pocos datos en población pediátrica. La presentación clínica varía desde dolor abdominal leve hasta complicaciones potencialmente mortales, como aquellas que comprometen el área mesentérica, siendo las más frecuentes la peritonitis, el infarto y la perforación intestinal. ${ }^{1,5} \mathrm{El}$ compromiso de otros órganos es un factor relevante al momento de considerar el diagnóstico. Frecuentemente, si su presentación es exclusivamente GI, puede imitar el debut de una enfermedad inflamatoria, tal como lo reportan De Carpi y col. ${ }^{6}$ En su publicación se describe el caso de un adolescente que, luego de presentar dolor abdominal, elevación de reactantes de fase aguda e inflamación íleo-colónica en la endoscopía, fue diagnosticado en primera instancia con enfermedad de Crohn. Luego de agregarse una crisis convulsiva, hipertensión arterial y constatarse lesiones isquémicas cerebrales en la RNM, el diagnóstico final fue de periarteritis nodosa. Por otro lado, la concomitancia o aparición posterior de síntomas que comprometen otros órganos pueden ayudar al diagnóstico diferencial, tal como sucedió con nuestra paciente. La presencia del compromiso renal nos proporcionó indicios sobre la presencia de vasculitis subyacente como causante de la hemorragia diges-
Figura 3. Anatomía patológica de duodeno, vasculitis focal en vasos capilares.

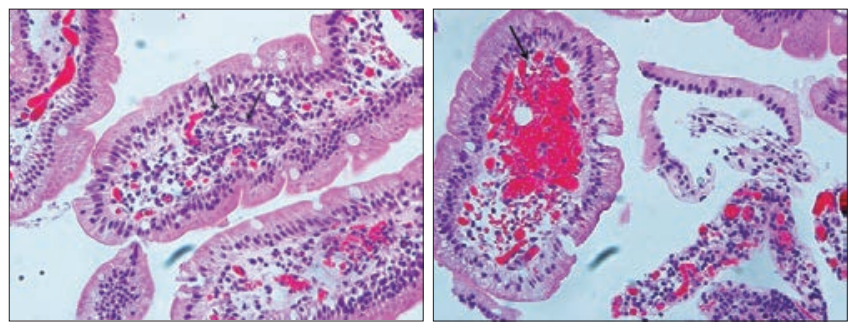

tiva (HD). La angiografía visceral selectiva parece jugar un papel relevante cuando existen dudas diagnósticas. Una revisión pediátrica realizada en dos centros de referencia de Londres observó que en diez pacientes con debut GI y posterior diagnóstico de vasculitis sistémica, la angiografía fue sugerente del trastorno en todos ellos. ${ }^{7}$ El mapeo de otros territorios por angio RMN en el contexto de una vasculitis inusual en pediatría parece ser una herramienta valiosa. Debido a la escasa especificidad de los hallazgos endoscópicos y anatomopatológicos, el diagnóstico definitivo de vasculitis GI es difícil de establecer. La presentación endoscópica más frecuente en este tipo de patologías son las lesiones de tipo ulcerosas, así como el eritema y hematoma de la mucosa. ${ }^{8}$ Las biopsias endoscópicas, por su parte, parecen tener una baja sensibilidad para diagnosticar vasculitis, ya que la enfermedad es irregular, incluso cuando la apariencia endoscópica es sugestiva. En estos casos, la histología revela cambios inflamatorios crónicos en la mucosa, ocasionando, como ya se mencionó, interpretaciones erróneas. ${ }^{7}$ El compromiso macroscópico y microscópico en nuestro caso presentado fue categórico, con lesiones endoscópicas de aspecto vasculítico y confirmación histopatológica. Cabe destacar, que cuando un proceso vasculítico involucra el intestino, los cambios son predominantemente submucosos y las biopsias realizadas suelen ser más superficiales. Una 
revisión en pacientes adultos observó que durante los tres primeros meses de diagnosticada una vasculitis de pequeños y medianos vasos, la HD fue una presentación poco frecuente $(20 \%)$, encontrándose signos histológicos de vasculitis solo en el 4,8\% de los casos. ${ }^{1}$ En nuestra paciente se realizaron un total de siete biopsias, de las cuales una de ellas fue categórica para compromiso vascular. Si bien se constató friabilidad de la mucosa al momento de la toma de muestra, no tuvimos complicaciones una vez finalizado el procedimiento.

\section{Conclusión}

La vasculitis del tracto GI es una condición infrecuente, que puede presentarse en forma exclusiva a nivel vascular o comprometiendo a otros órganos. En nuestra paciente se diagnosticó luego de haberse generado un compromiso de órgano blanco, situación que nos proporcionó una rápida sospecha diagnóstica. Si bien las biopsias endoscópicas parecen tener una baja sensibilidad diagnóstica por el carácter superficial de la muestra, consideramos importante realizarlas en forma prudente y adecuada en todos los sectores sugestivos, ya que la información proporcionada es de suma relevancia. En conclusión, hemos presentado un compromiso de órgano poco frecuente en un paciente pediátrico con vasculitis ANCA-p. Creemos adecuado sospecharlo ante todo compromiso intestinal en el que no sea claro el proceso inflamatorio subyacente, más aún cuando compromete otros órganos. En esta instancia, la angiografía visceral selectiva puede ser de gran utilidad, ya que puede mostrar signos de afectación vascular. Como hemos comentado, estas patologías no están exentas de complicaciones, por lo que un reconocimiento temprano y un tratamiento oportuno son las claves para abordar estos pacientes.

Agradecimientos. Dra. Ferraris Verónica y Dra. Pineda Laura en su aporte a la recolección de datos.

Dra. De la Iglesia Paola en la descripción de la imagen anatomopatológica.

\section{Conflicto de intereses. Ninguno a declarar.}

Consentimiento para la publicación. Se obtuvo el consentimiento informado por escrito del paciente o sus padres, tutor o familiar, para la publicación de los datos y/o imágenes clínicas en beneficio de la ciencia. La copia del formulario de consentimiento se encuentra disponible para los editores de esta revista.
Propiedad intelectual. Los autores declaran que los datos y las figuras presentes en el manuscrito son originales y fueron realizados en las instituciones a las que pertenecen.

\section{Aviso de derechos de autor}

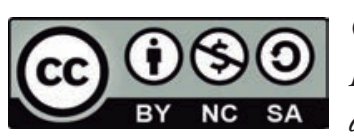

(C) 2021 Acta Gastroenterológica Latinoamericana. Este es un artículo de acceso abierto publicado bajo los términos de la Licencia Creative Commons Attribution (CC BY-NC-SA 4.0), la cual permite el uso, la distribución y la reproducción de forma no comercial, siempre que se cite al autor y la fuente original.

Cite este artículo como: Tagliaferro $G J$, Busoni V, Bertinotti $M C$ y col. Compromiso gastrointestinal inusual en paciente pediátrico con vasculitis asociada a anticuerpos anticitoplasma de neutrófilos. Reporte de un caso. Acta Gastroenterol Latinoam. 2021;51(4):437-0. https://doi. org/10.52787/USCW3486

\section{Referencias}

1. Pagnoux C, Mahr A, Cohen P, Guillevin L. Presentation and outcome of gastrointestinal involvement in systemic necrotizing vasculitides: analysis of 62 patients with polyarteritis nodosa, microscopic polyangiitis, Wegener granulomatosis, Churg-Strauss syndrome, or rheumatoid arthritis-associated vasculitis. Medicine. 2005;84: 115-28.

2. Calatroni M, Oliva E, Gianfreda D, Gregorini G, Allinovi M, Ramirez GA, et al. ANCA-associated vasculitis in childhood: recent advances. Ital J Pediatr. 2017;43:46.

3. DiMeo DE, Ferguson PJ, Bishop WP. An Unusual Intestinal Presentation of C-ANCA/PR-3 Positive Vasculitis in a Child. Journal of Pediatric Gastroenterology and Nutrition. 2005:368-70. doi:10.1097/01.mpg.0000155368.46841.21

4. Özçelik G, Sönmez HE, Şahin S, Özağarı A, Bayram MT, Çiçek $\mathrm{RY}$, et al. Clinical and histopathological prognostic factors affecting the renal outcomes in childhood ANCA-associated vasculitis. Pediatr Nephrol. 2019;34:847-54.

5. Kronzer VL, Larson DP, Crowson CS, Warrington KJ, Ytterberg SR, Makol A, et al. Occurrence and aetiology of gastrointestinal perforation in patients with vasculitis. Clin Exp Rheumatol. 2019;37 Suppl 117:32-9.

6. De Carpi JM, Castejón E, Masiques L, Vilar P, Antón J, Varea V. Gastrointestinal involvement in pediatric polyarteritis nodosa. J Pediatr Gastroenterol Nutr. 2007;44: 274-8.

7. Brogan PA, Malik M, Shah N, Kilday JP, Ramsay A, Shah V, et al. Systemic vasculitis: a cause of indeterminate intestinal inflammation. J Pediatr Gastroenterol Nutr. 2006;42:405-15.

8. Hokama A, Kishimoto K, Ihama Y, Kobashigawa C, Nakamoto M, Hirata T, et al. Endoscopic and radiographic features of gastrointestinal involvement in vasculitis. World J Gastrointest Endosc. 2012;4:50-6. 